\title{
Simultaneidade de fatores de risco cardiovascular modificáveis na população adulta de Salvador (BA), Brasil ${ }^{1}$
}

\author{
Ines Lessa, ${ }^{2}$ Maria Jenny Araújo, ${ }^{3}$ Lucélia Magalhães, ${ }^{4}$ \\ Naomar de Almeida Filho, ${ }^{2}$ Estela Aquino ${ }^{2}$ e Maria Cecília R. Costa ${ }^{5}$
}

Cómo citar Lessa I, Araújo MJ, Magalhães L, Almeida Filho N de, Aquino E, Costa MCR. Simultaneidade de fatores de risco cardiovascular modificáveis na população adulta de Salvador (BA), Brasil. Rev Panam Salud Publica. 2004;16(2):131-7.

RESUMO Objetivo. Estimar a freqüência da simultaneidade de fatores de risco cardiovascular modificáveis, com e sem inclusão da hipertensão arterial, em uma população miscigenada.

Método. Estudo transversal realizado em 1298 adultos com idade $\geq 20$ anos na Cidade de Salvador, Brasil, em 2000. Foram incluídos oito fatores de risco cardiovascular modificáveis, considerados em qualquer combinação: colesterol total $\geq 240 \mathrm{mg} / \mathrm{dL}$; colesterol das lipoproteínas de alta densidade $(H D L-c)<40 \mathrm{mg} / \mathrm{dL} ;$ triglicerídeos $\geq 200 \mathrm{mg} / \mathrm{dL} ;$ glicemia $\geq 126 \mathrm{mg} / \mathrm{dL}+$ diabetes controlado; indice de massa corporal $\geq 25 \mathrm{~kg} / \mathrm{m}^{2}$, cintura $\geq 102 \mathrm{~cm}$ (homens) $e \geq 88 \mathrm{~cm}$ (mulheres), tabagismo e alcoolismo. Os resultados foram estratificados de acordo com o número de fatores de risco simultâneos (de zero a cinco ou mais; e dois ou mais). Os dados foram analisados em termos de proporções estimadas e intervalo de confiança de 95\% (IC95\%), com e sem inclusão da hipertensão arterial (critério JNC-VI), razões entre proporções e qui-quadrado para proporções como medida de associação.

Resultados. Entre os homens (41,4\% dos participantes), 7,5\% (IC95\%: 2,5 a 9,7) não apresentaram fatores de risco; 68,8\% (IC95\%: 65,0 a 72,8) apresentaram dois ou mais fatores de risco, excluída a hipertensão. Quando a hipertensão foi incluída, 73,4\% (IC95\%: 69,7 a 77,1) apresentaram dois ou mais fatores de risco. Entre as mulheres, $11,6 \%$ não apresentaram fatores de risco. A presença de dois ou mais fatores de risco, excluída a hipertensão, foi observada em $67,7 \%$ (IC95\%: 64,8 a 71,4). Após inclusão da hipertensão, 71,7\% (IC95\%: 68,5 a 74,9) das mulheres apresentaram dois ou mais fatores de risco. Foram observadas diferenças significativas entre presença de dois ou mais fatores de risco para homens com até 4 anos de estudo versus homens com 5 a menos de 11 anos de estudo ( $<<0,05)$; mulheres com até 4 anos de estudo versus 5 a menos de 11 anos de estudo; mulheres com até 4 anos de estudo versus 11 ou mais anos de estudo $(\mathrm{P}<$ $0,01)$; e para mulheres negras versus brancas ( $\mathrm{P}<0,01)$.

Conclusão. A elevada proporção de múltiplos fatores de risco cardiovascular em Salvador, incluindo-se ou não a hipertensão, especialmente na população de baixa escolaridade e em pessoas negras, sugere a necessidade de estratégias sociais abrangentes para reduzir as desigualdades sociais, promover a saúde, e facilitar o tratamento de fatores de risco cardiovascular.

Palavras-chave

Alcoolismo, dislipidemia, diabetes mellitus, hipertensão, índice de massa corporal, peso corporal, tabagismo.

\footnotetext{
Trabalho financiado pelo Ministério da Saúde, Centro Nacional de Epidemiologia, Brasil. Ines Lessa, Naomar de Almeida Filho e Estela Aquino são pesquisadores do Conselho Nacional de Desenvolvimento Científico e Tecnológico (CNPq).

2 Universidade Federal da Bahia (UFBa), Instituto de Saúde Coletiva, Salvador (BA), Brasil. Corres-
}

pondência para Ines Lessa no seguinte endereço: Rua Barachísio Lisboa 3, Horto Florestal, CEP 40295-120, Salvador, BA, Brasil. Fone: +55-71-3343232; e-mail: ines@lessa.org, inlessa@terra.com.br, inlessa@ufba.br
3 UFBa, Escola de Enfermagem.

4 Secretaria de Saúde do Estado da Bahia, Salvador (BA), Brasil.

5 UFBa, Escola de Nutrição. 
As projeções referentes às doenças cardiovasculares (DCV) indicam sua permanência como primeira causa de morte no mundo ainda por décadas, estimando-se que, em 2025, entre 80 e $90 \%$ dos casos ocorrerão nos países de baixa e média renda (1). São comuns taxas de prevalência elevadas dos clássicos fatores de risco para as DCV (sobrepeso e obesidade, tabagismo, elevação de lipídios séricos, hipertensão arterial e diabetes mellitus), com variações em magnitude em diferentes espaços geográficos e sociais (2). Alguns desses fatores tendem a pequeno declínio, enquanto outros tendem à estabilidade ou ascensão $(2,3)$, como no caso do tabagismo entre jovens e da obesidade em crianças e adultos (1). A obesidade, inclusive, passou a se comportar como epidemia com tendência a pandemia, como resultado de mudanças sociais e culturais nos padrões de alimentação e atividade física $(1,4,5)$.

As estratégias para prevenção, tratamento e controle das DCV centradas em fatores de risco isolados ou em estimativas dos riscos relativos não apresentaram os resultados esperados (6). Assim, a partir dos anos 1990, a prevenção das DCV passou a enfocar o risco cardiovascular global, que é baseado no conjunto de fatores de risco cardiovascular (FRCV) simultaneamente presentes em um mesmo indivíduo. O risco global, ou risco absoluto (incidência) (1, 7-10), vem sendo estimado pelo algoritmo para eventos futuros da doença coronariana, construído com base na coorte de Framingham, que leva em consideração idade, sexo, níveis séricos de colesterol das lipoproteínas de alta densidade (highdensity lipoprotein cholesterol, HDL-c), pressão arterial sistólica, presença de diabetes e tabagismo $(11,12)$, ou por adaptações realizadas por outros investigadores $(9,10)$. Conquanto as projeções para eventos futuros sejam individualizadas, as taxas de prevalência populacionais de agrupamentos de FRCV são elevadas (13-15), devendo toda a comunidade ser contemplada com medidas de promoção à saúde (12), com maior ênfase nos grupos de maior risco. Preferencialmente, as estratégias de prevenção devem ser dire- cionadas para o conjunto das doenças cardiovasculares dependentes dos fatores de risco acima referidos (11) e de outros FRCV.

Dentre as DCV, as cerebrovasculares são a primeira causa de morte no Brasil. A insuficiência cardíaca, por sua vez, é responsável pela maior taxa de hospitalização, enquanto a prevalência da hipertensão arterial (HA), medida a partir da década de 1990 em alguns locais, variou entre 24 e $42 \%(16,17)$. Também é elevada a prevalência de múltiplos FRCV em localidades das regiões Sul (18) e Sudeste (19) do Brasil, que correspondem às áreas mais desenvolvidas do país. É importante ampliar o conhecimento sobre o impacto dos FRCV em áreas menos desenvolvidas e com características sócio-culturais diversas. Assim, o objetivo deste estudo foi estimar a prevalência de FRCV mutáveis simultâneos na população fortemente miscigenada da Cidade de Salvador, Região Nordeste do Brasil, e o impacto determinado pela inclusão da HA entre esses fatores.

\section{MATERIAIS E MÉTODOS}

Trata-se de estudo transversal, realizado em subgrupo de 1298 dentre 2298 adultos com idade $\geq 20$ anos que participaram do Projeto Monitoramento das Doenças Cardiovasculares e do Diabetes (MONIT) em Salvador (BA), Brasil, 2000 (20). Para o MONIT, a amostragem por conglomerados foi realizada em três estágios. Em primeiro lugar, os setores censitários de oito das 10 bacias hidrográficas da cidade, com características sócio-demográficas semelhantes, foram agrupados em 108 áreas de pesquisa, que foram classificadas por nível socioeconômico alto, misto e baixo. Ao todo, as áreas continham 16592 domicílios, com aproximadamente 83000 habitantes com 20 anos ou mais. Foram sorteadas por amostra probabilistica 37 áreas, proporcionalmente ao número de setores de cada nível socioeconômico. No segundo estágio foram sorteados 1540 domicílios por amostra sistemática (intervalo $=10$ ). Concordaram em participar 1258 famílias
$(81,7 \%)$ residentes em 63 setores censitários. No terceiro estágio foram sorteados os participantes, no máximo dois por domicílio, um de cada sexo. A metodologia da parte básica do projeto original foi recentemente descrita em detalhe em outra publicação (21). O subgrupo dos 1298 indivíduos aqui analisados correspondeu a $56,5 \%$ dos 2297 participantes do estudo global e a $84,0 \%$ dos 1546 que compareceram para completar o protocolo. As perdas se deram pela ausência das medidas de peso e altura em 141 indivíduos que haviam coletado sangue e pelo extravio de 107 protocolos completos antes da digitação.

Todos os participantes do projeto original responderam a questionário sobre FRCV e presença de HA ou diabetes, e comprovaram o tratamento pela apresentação da medicação, embalagem ou prescrição médica. $\mathrm{O}$ controle dessas doenças foi confirmado pelos exames realizados pelo projeto. Foram efetuadas: seis medidas da pressão arterial em dois blocos diferentes de três medidas cada uno com intervalo aproximado de 10 minutos entre os blocos, e medidas da circunferência da cintura. Esses procedimentos foram realizados por técnicas padronizadas, seguindo todas as recomendações usuais em epidemiologia para a medida da pressão arterial e da circunferência. $\mathrm{O}$ esfigmomanômetro utilizado foi o Omron HEM 705 CP, modelo eletrônico, testado e aprovado pela British Hypertension Society (22), cujas maiores vantagens são: a) facilidade de treinamento e de padronização; b) eliminação de vieses de mensuração decorrentes da visão, audição e atenção; c) impossibilidade de opção por dígitos terminais pelos entrevistadores; e d) não interferência na velocidade de inflação/deflação do manguito. A circunferência foi medida com fita metálica rígida.

No centro de saúde ou serviço médico de cada área, a equipe do projeto procedeu a: a) medida da altura e do peso para o cálculo do índice de massa corporal (IMC), usando equipamentos padronizados e aferidos periodicamente pelo Instituto Nacional de Metrologia, Normalização e Qualidade In- 
dustrial (Inmetro); b) coleta da amostra de sangue pós-jejum de $12 \mathrm{~h}$ para exames bioquímicos, entre as quais o colesterol total, o HDL-c, os triglicerídeos e a glicemia. Todos os procedimentos foram realizados em um mesmo laboratório por métodos e equipes padronizados. O controle de qualidade das aferições antropométricas e da pressão arterial foi realizado por estimativa de médias e medidas de dispersão inter e intra entrevistadores, sob a responsabilidade de dois dos co-autores (MJA e LM) e de uma nutricionista.

Oito variáveis foram selecionadas como FRCV mutáveis (resultantes de hábitos e costumes indesejáveis que podem ser modificados, minimizados ou eliminados por intervenções educacionais e preventivas) para combinações neste estudo e são abaixo relacionadas, com seus critérios de anormalidade:

- sobrepeso (inclusive obesidade): IMC (peso/altura ${ }^{2}$ ) $\geq 25 \mathrm{~kg} / \mathrm{m}^{2}$;

- obesidade central: circunferência da cintura $\geq 88 \mathrm{~cm}$ para mulheres e $\geq 102 \mathrm{~cm}$ para os homens, independentemente da presença de obesidade generalizada;

- colesterol total $\geq 240 \mathrm{mg} / \mathrm{dL}$ pelo método enzimático de Trinder;

- HDL-c < $40 \mathrm{mg} / \mathrm{dL}$ pelo método Labtest;

- triglicerídeos $\geq 200 \mathrm{mg} / \mathrm{dL}$ pelo método de Soloni modificado;

- glicemia $\geq 126 \mathrm{mg} / \mathrm{dL}+$ diagnóstico comprovado de diabetes, em tratamento, e glicemia de jejum normal (método enzimático de Trinder);

- tabagismo: fumante atual, qualquer número de cigarros por dia;

- consumo de álcool em finais de semana com embriaguez freqüente ou consumo diário, com ou sem embriaguez.

Cada participante foi classificado quanto ao número de FRCV simultaneamente presentes, e em agrupamentos de dois ou mais fatores de risco a cinco ou mais fatores de risco, sempre em qualquer combinação entre eles. Além desses oito FRCV relacionados à epidemiologia da HA, das doenças cerebrovasculares, da doença arterial co- ronariana e da vasculopatia periférica, a HA entrou como um nono FRCV mutável para estimativa do seu impacto na proporção da contribuição dos FRCV para as três últimas situações referidas, conforme objetivo. A presença de HA foi definida conforme o critério JNC-VI: pressão arterial sistólica $\geq 140$ $\mathrm{mmHg}$ ou pressão arterial diastólica $\geq 90 \mathrm{mmHg}+$ casos de hipertensão conhecida, com pressão arterial controlada, comprovada pelo tratamento em uso. A HA foi o único FRCV selecionado que entrou sistematicamente em todos os estratos de FRCV (exceto no estrato relativo à ausência de fatores de risco).

Outras variáveis independentes incluídas não são fatores mutáveis: idade, sexo, raça (variáveis biológicas) e escolaridade (variável social). Em razão da forte miscigenação racial no país, qualquer critério para definição de raça é questionável, de modo que a raça foi auto-definida pela cor da pele (branca, parda ou negra), conforme a nomenclatura oficial utilizada nos censos demográficos. A escolaridade foi estratificada em baixa (analfabetos + escolarizados até a quarta série do ensino fundamental); média (quinta série do ensino fundamental até terceira série incompleta do ensino médio) e alta (ensino médio completo + superior incompleto ou completo, $\geq 11$ anos completos de escola).

A análise foi descritiva por estimativa da proporção do número de FRCV e seus intervalos de confiança de 95\% (IC95\%), antes e após a inserção da HA. Foram também calculadas as diferenças absoluta e relativa entre as proporções referidas. Diferenças estatísticas entre as proporções de dois ou mais ou cinco ou mais FRCV foram estimadas para o mesmo sexo e entre os sexos pelo teste do qui-quadrado $\left(\chi^{2}\right)$ para proporções. Outras associações também foram estimadas pelo $\chi^{2}$. As razões de proporções (RP) foram usadas para discriminar a predominância das características sócio-demográficas por sexo.

O projeto foi aprovado pelo Comitê de Ética Médica do Conselho Regional de Medicina do Estado da Bahia e todos os participantes assinaram termo de consentimento informado.

\section{RESULTADOS}

Dos 1298 participantes, 537 (41,4\%) eram homens, correspondendo a $51,3 \%$ da amostra masculina original ( $\mathrm{n}=1047$ ). As 761 (58,6\%) mulheres representaram $60,9 \%$ da amostra feminina de origem $(n=1250)$. Os participantes do subgrupo foram semelhantes à amostra de origem quanto a idade, raça, escolaridade e outras variáveis.

A tabela 1 apresenta as informações relativas às variáveis biológicas e à escolaridade para ambos os sexos. A distribuição etária aproxima-se entre homens e mulheres, excetuando-se a distribuição para homens no grupo mais jovem e para mulheres no mais idoso $(P<0,05)$. Excluída a alta escolaridade, 1,6 vezes mais elevada nos homens, todas as RP para baixa e média escolaridade foram semelhantes entre os sexos (tabela 1). A tabela 2 mostra a proporção de FRCV em homens e mulheres com e sem a presença de HA. Em qualquer dos agrupamentos analisados, a proporção de FRCV simultâneos, sem inclusão da HA, foi semelhante entre os sexos $(P>0,05)$. Após a inclusão da HA o agrupamento de quatro fatores de risco foi desfavorável aos homens $(P<0,05)$ e o de cinco fatores de FRCV simultâneos foi desfavorável às mulheres $(P<0,05)$.

A HA isolada, na ausência de qualquer dos oito fatores de risco, foi observada em cinco homens e sete mulheres $(0,9 \%$ dos participantes de cada sexo), correspondendo a 2,0\% dos casos de HA em homens e 2,1\% nas mulheres.

$\mathrm{O}$ número de fatores de risco aumentou com a idade. Nas figuras 1 e 2 encontram-se as variações nas proporções de FRCV (zero a cinco ou mais) por grupo etário em cada sexo. Nota-se decréscimo com a idade para presença de apenas um fator de risco e aumento considerável das proporções de dois a quatro fatores de risco simultâneos para os homens. Para mulheres, a proporção de apenas um fator de risco decresceu até a faixa dos 50 aos 59 anos, aumentando as proporções para as demais combinações. Em ambos os sexos as proporções de 
TABELA 1. Características sócio-demográficas dos adultos participantes do estudo sobre múltiplos fatores de risco cardiovascular, Salvador (BA), Brasil, 2002

\begin{tabular}{|c|c|c|c|c|c|}
\hline \multirow[b]{2}{*}{ Variáveis } & \multicolumn{2}{|c|}{ Homens } & \multicolumn{2}{|c|}{ Mulheres } & \multirow{2}{*}{$\begin{array}{c}\text { Razão de freqüência } \\
\text { (Homens/Mulheres) }\end{array}$} \\
\hline & No & $\%$ & No & $\%$ & \\
\hline Sexo & 537 & 41,4 & 761 & 58,6 & 0,7 \\
\hline \multicolumn{6}{|l|}{ Idade (anos) } \\
\hline 20 a 29 & 144 & 26,8 & 168 & 22,1 & 1,2 \\
\hline 30 a 39 & 130 & 24,2 & 215 & 28,3 & 0,9 \\
\hline 40 a 49 & 141 & 26,3 & 178 & 23,4 & 1,1 \\
\hline 50 a 59 & 66 & 12,3 & 100 & 13,1 & 0,9 \\
\hline 60 a 69 & 38 & 7,1 & 59 & 7,8 & 0,9 \\
\hline 70 ou mais & 18 & 3,4 & 41 & 5,4 & 0,6 \\
\hline \multicolumn{6}{|l|}{ Escolaridade $^{a}$} \\
\hline Baixa & 236 & 44,3 & 377 & 49,7 & 0,9 \\
\hline Média & 273 & 51,2 & 360 & 47,5 & 1,1 \\
\hline Alta & 24 & 4,5 & 21 & 2,8 & 1,6 \\
\hline \multicolumn{6}{|c|}{ Raça/cor da pele } \\
\hline Branca & 133 & 24,9 & 202 & 26,8 & 0,9 \\
\hline Parda & 250 & 46,8 & 341 & 45,3 & 1,0 \\
\hline Negra & 151 & 28,3 & 210 & 27,9 & 1,0 \\
\hline
\end{tabular}

a Escolaridade baixa: analfabetos + escolarizados até a quarta série do ensino fundamental; média: quinta série do ensino fundamental até a terceira série incompleta do ensino médio; alta: ensino médio completo + superior incompleto ou completo, $\geq 11$ anos completos de escola.

FRCV simultâneos foram elevadas entre os jovens.

Associação estatisticamente significativa foi observada para mulheres negras em relação às brancas $(P<0,01)$; para homens e mulheres com baixa em relação à média escolaridade $(P<$ $0,05)$; e para mulheres com baixa escolaridade em relação às de alta escolaridade $(P<0,01)$ (tabela 3$)$.

\section{DISCUSSĀO}

A população de procedência dos participantes caracteriza-se como de elevado risco cardiovascular. As proporções de dois ou mais FRCV de $69,0 \%$ para homens e de $68,1 \%$ para mulheres superaram os valores referidos de $31 \%$ entre 18 e 59 anos e de $42,0 \%$ em idades $\geq 60$ anos para hiper- tensos de Bambuí, na Região Sudeste (19), e os 39,0\% descritos para Porto Alegre, na Região Sul do Brasil (18). As divergências entre a magnitude dessas taxas de prevalência podem ser atribuídas ao maior número de fatores de risco estudados em Salvador. Quanto maior o número de variáveis usadas para agrupamentos, tanto maior o número de combinações possíveis e maiores as chances de detecção de freqüências mais elevadas para dois ou mais FRCV ou, isoladamente, para cada grupo de fatores de risco.

Dos cinco FRCV estudados em Porto Alegre, quatro foram auto-referidos e nenhum metabólico (18). No presente estudo, só em relação aos fatores lipídicos, foram incluídos o colesterol total, o HDL-c e os triglicerídeos. Combinações entre os próprios fatores lipídicos devem ter sido freqüentes, dado que a hipercolesterolemia, isoladamente, foi detectada em $31 \%$ da amostra, mesmo que o HDL-c baixo e a hipertrigliceridemia não tenham apresentado taxas de prevalência exageradamente elevadas (20).

Freqüentemente, os fatores metabólicos acompanham a HA e associamse às demais doenças cardiovasculares ateroscleróticas $(10,16)$. Os fatores metabólicos mais comuns em estudos internacionais $(9,10,13,15)$ foram também usados neste estudo. Geral-

TABELA 2. Proporção de fatores de risco cardiovascular simultâneos ${ }^{a}$ com e sem inclusão da hipertensão arterial, Salvador (BA), Brasil, 2000

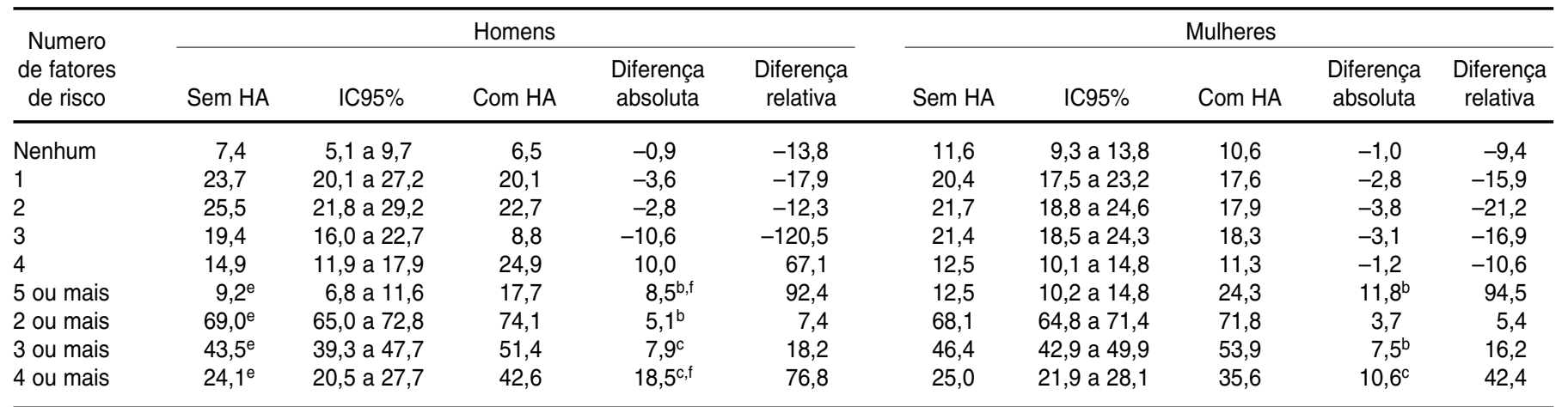

a Simultaneidade = qualquer combinação entre: sobrepeso, obesidade central, hipercolesterolemia, baixo HDL-c, hipertrigliceridemia, hiperglicemia, tabagismo e consumo excessivo de álcool. $\mathrm{HA}=$ hipertensão arterial.

b $P<0,05$ para comparações do mesmo sexo.

c $P<0,01$ para comparações do mesmo sexo.

d $P<0,001$ para comparações do mesmo sexo.

e $P>0,05$ para comparações entre os sexos.

f $P<0,05$ para comparações entre os sexos. 
FIGURA 1. Freqüência de múltiplos fatores de risco cardiovascular por idade, sexo masculino, Salvador (BA), Brasil, 2000

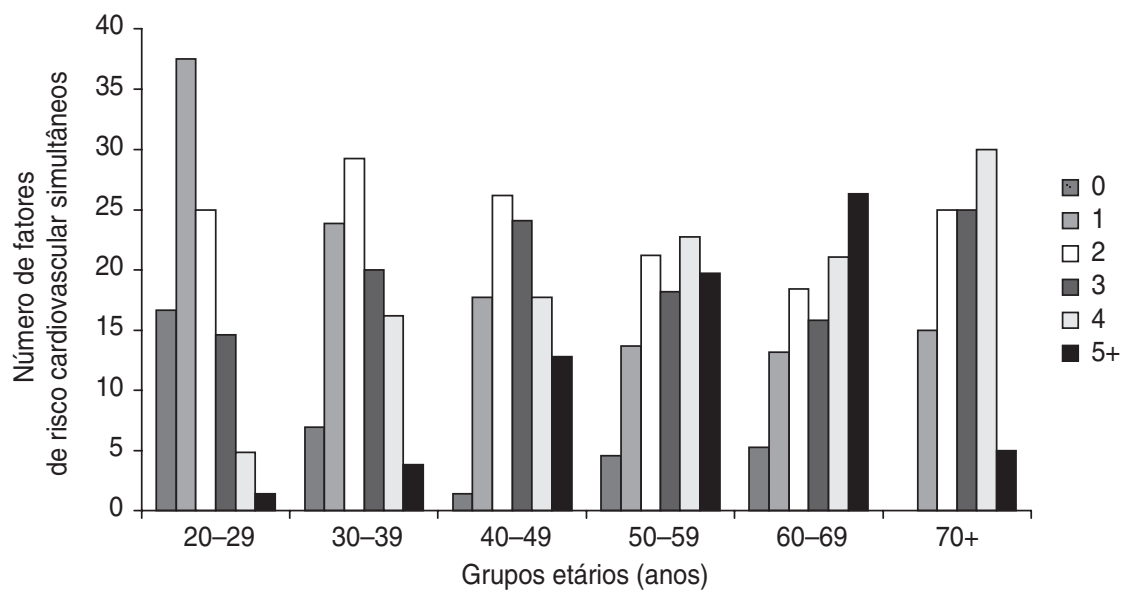

mente, a hipertensão é incluída como um dos componentes das combinações de múltiplos fatores de risco cardiovascular, diferente do enfoque deste estudo. Sabe-se que a HA, o diabetes, a obesidade e as dislipidemias podem ser parcialmente prevenidos por intervenções sobre fatores de risco comportamentais, com resultados favoráveis sobre suas taxas de prevalência na população. Contudo, pela elevada prevalência da HA no Brasil e pelo elevado custo social de suas conseqüências, a medida da freqüência de múltiplos FRCV com e sem a inclusão da HA é social e econo- micamente relevante para estratégias preventivas.

Quando a HA foi deliberadamente incluída nos grupos como um nono fator de risco, diminuiu a já baixa proporção do grupo representado pela ausência de FRCV e ocorreu um deslocamento de pessoas de grupos com menor número de fatores de risco para os grupos subseqüentes, com acentuado impacto naqueles que já apresentavam quatro, cinco, ou mais FRCV. A presença da HA em concomitância com outros FRCV aumentou a proporção de dois ou mais fatores de risco, agravando o perfil de risco car-

FIGURA 2. Freqüência de múltiplos fatores de risco cardiovascular por idade, sexo feminino, Salvador (BA), Brasil, 2000

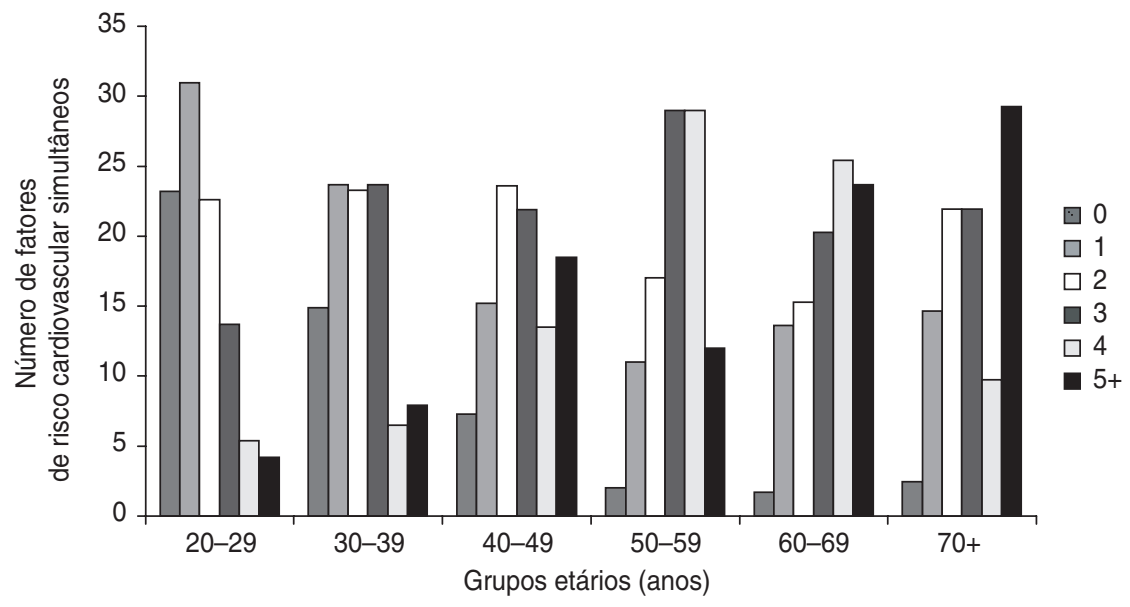

diovascular em ambos os sexos, a partir de dois ou mais FRCV para os homens e de três ou mais FRCV para as mulheres. No entanto, na comparação entre os sexos, as proporções de dois ou mais e de três ou mais FRCV incluindo HA não diferiram estatisticamente $(P>0,05)$. Foi observada diferença estatística para quatro ou mais (desfavorável às mulheres) e cinco ou mais FRCV (desfavorável aos homens). Portanto, é essencial enfatizar a atuação sobre os FRCV de modo global e o compromisso com o diagnóstico precoce da hipertensão na atenção básica à saúde.

A freqüência da HA isolada, sem qualquer FRCV associado foi muito baixa, não tendo alcançado $1 \% \mathrm{em}$ cada sexo. Por outro lado, os $2 \%$ de hipertensão na ausência de FRCV sugerem a possibilidade de hipertensão secundária ou dependente de outro fator de risco não analisado, a exemplo dos estressores sociais não trabalhados no estudo. Raça e baixa escolaridade, mesmo que relacionadas aos possíveis estressores sociais, não foram aqui exploradas como tais.

Raça negra, baixa escolaridade e baixo nível socioeconômico são descritos como de elevado risco cardiovascular na literatura internacional $(2,4)$. Para essas variáveis, as freqüências observadas em Salvador foram, de modo geral, mais altas. Isoladamente, apresentaram associações positivas, estatisticamente significativas, com dois ou mais FRCV, excetuando-se a significância estatística para a raça no sexo masculino. A predominância da aglomeração dos fatores nos segmentos sociais de pardos e negros e no de baixa escolaridade confirma as observações de Duncan et al. (18) em Porto Alegre. Esses grupos sociais foram identificados como sendo os de maior prevalência para FRCV estudados isoladamente em algumas localidades do país $(23,24)$.

As freqüências de dois ou mais FRCV em Salvador ultrapassaram os $54,3 \%$ referidos para homens e os $21,3 \%$ para mulheres na China (13), assim como os 28 a $30 \%$ referidos para mulheres nos Estados Unidos (14). Para três ou mais fatores de risco, as proporções detectadas em Salvador, de $43,5 \%$ nos 
TABELA 3. Proporção do número de fatores de risco cardiovascular simultâneos por sexo, cor da pele e escolaridade, Salvador (BA), Brasil, 2000

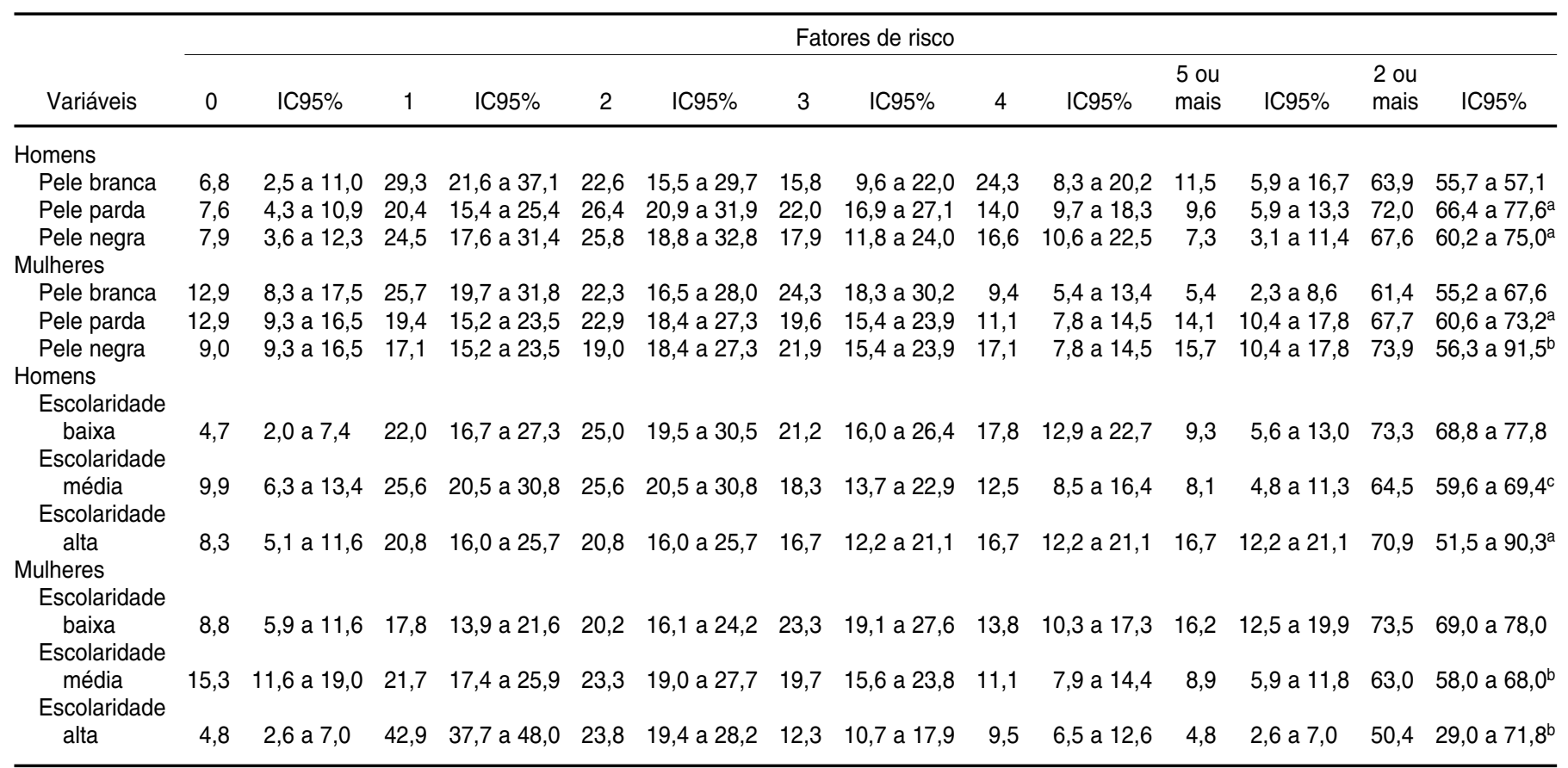

${ }^{\mathrm{a}} \chi^{2}$ para proporções: $P<0,05$

b $\chi^{2}$ para proporções: $P<0,01$

${ }^{c} \chi^{2}$ para proporções: $P<0,05$

homens e de $46,4 \%$ nas mulheres, foram inferiores aos $59 \%$ descritos por Hoffman e Cubeddou para espanhóis em análise de 10 fatores de risco (15).

Não se pretendeu neste estudo discriminar domínios com FRCV específicos predominantes. No entanto, os oito fatores de risco selecionados, além da HA, são internacionalmente reconhecidos como sendo da maior relevância para as doenças cardiovasculares, de modo que, em qualquer combinação, são importantes para decisões preventivas.

Razões econômicas ou ambientes sócio-culturais são mencionados como uma possível explicação para as am- plas variações geográficas internacionais da prevalência de FRCV isolados ou múltiplos em distintas etnias (2), e como responsáveis pelas elevadas freqüências das doenças cardiovasculares nos países em desenvolvimento (5). Em Salvador, cidade com mais de 2500000 habitantes, $70 \%$ miscigenados, e elevada freqüência de baixa escolaridade e de baixa renda, é crítico o perfil de risco cardiovascular da população adulta estudada. Uma melhoria indiscriminada do nível de escolaridade da população provavelmente seria acompanhada de melhoria no nível socioeconômico e poderia minimizar a in- fluência racial na elevada prevalência de multiplicidade de FRCV. Paralelamente, as estratégias com foco no risco cardiovascular global, obrigatórias para os indivíduos sob assistência, devem ser implementadas de forma extensiva e continuada para a população, além de monitoradas. Ênfase deve ser dada a atividades educativas e informativas que levem em conta as diferenças sociais e culturais. Os benefícios não serão apenas cardiovasculares, uma vez que os fatores de risco considerados são relevantes também para outras doenças, como o diabetes (25), em acelerada expansão mundial.

\section{REFERÊNCIAS}

1. Bonow RO, Smaha LA, Smith Jr SC, Mensah $\mathrm{GA}$, Lenfant $\mathrm{C}$. The international burden of cardiovascular disease: responding to the emerging global epidemic. [Special Report, World Heart Day 2002]. Circulation. 2002; 106(13):602-1606.

2. Yusuf S, Reddy S, Ônpuu S, Anand S. Global burden of cardiovascular diseases. Part II: Variations in cardiovascular disease by spe- cific ethnic groups and geographic regions and prevention strategies. Circulation. 2001; 104(23):2855-64.

3. Arnett DK, McGovern PG, Jacobs DR, Shahar E, Duval S, Blackburn H, et al. Fifteen-year trends in cardiovascular risk factors (19801982 through 1995-1997). The Minnesota heart survey. Am J Epidemiol. 2002;156(10): 929-35.
4. Hahn RA, Heath GW, Chang MH. Cardiovascular disease risk factors and preventive practices among adults — United States, 1994: a behavioral risk factor atlas. Behavioral Risk Factor Surveillance System State Coordinators. MMWR CDC Surveill Summ. 1998;47(5): 35-69.

5. Levenson JW, Skerrett PJ, Gaziano MJ. Reducing the global burden of cardiovascular dis- 
ease: the role of risk factors. Prev Cardiol. 2002;5(4):188-99.

6. Jackson R. Guidelines on preventing cardiovascular disease in clinical practice [editorial]. Br Med J. 2000;320(7236):659-61.

7. Yusuf S, Ônpuu S, Anand S. The global epidemic of atheroesclerotic cardiovascular disease. Med Princ Pract. 2002;(Suppl 2):3-8.

8. Mahe I, Bergmann JF. Multifactorial cardiovascular risk. Press Med. 2000;29(1):47-54.

9. Wilson PWF, D'Agostino RB, Levy D, Belanger AM, Silbershatz H, Kannel WB. Prediction of coronary heart disease using risk factor categories. Circulation. 1998;97(18): 1837-47.

10. Kannel WB. Risk stratification in hypertension: new insights from Framingham Study. Am J Hypertens. 2000;13(1 pt 2):3S-10S.

11. Ramsay LE, Williams B, Johnathan G, MacGregor A, Poston L, Potter JF, et al. British Hypertension Society guidelines for hypertension management 1999: summary. Br Med J. 1999;319(7210):630-5.

12. Anderson KM, Odell PM, Wilson PW, Kannel WB. Cardiovascular disease risk profile. Am Heart J. 1991;121(1 Pt 2):293-8.

13. Wu D-M, Pai L, Chu N-F, Sung P-F, Lee M-S, Tsai, J-T, Hsu L-L, et al. Prevalence and clustering of cardiovascular risk factors among healthy adults in a Chinese population: The MJ Health Screening Center Study in Taiwan. Int J Obes Relat Met Disord. 2001;25(8): 1189-95.
14. Greenlund KJ, Gilles WH, Keenan NL, Croft JB, Casper ML, Matson-Koffman D. Prevalence of multiple cardiovascular disease risk factors among women in the United States, 1992 and 1995: the behavioral risk factor surveillance system. J Women Health. 1998;7(9): 1125-33.

15. Hoffmann IS, Arbeddu LX. Clustering of silent cardiovascular risk factors in apparently healthy Hispanics. J H Hypertens. 2002; 16(Suppl 1):S137-41.

16. Lessa I. Perfil das doenças cardiovasculares no Brasil. Em: Mion D, Nobre F. Risco cardiovascular global. $1^{\mathrm{a}}$ ed. Lemos Editorial: São Paulo; 1999. Pp. 15-28.

17. Lessa I. Epidemiologia da hipertensão arterial sistêmica e da insuficiência cardíaca no Brasil. Rev Bras Hipertens. 2001;8(4):383-92.

18. Duncan BB, Schmidt MI, Polanczkyk CA, Homirich CS, Rosa RS, Achutti AC. Fatores de risco para doenças não transmissíveis em área metropolitana do sul do Brasil. Prevalência e simultaneidade. Rev Saude Publica. 1993; 27(1):43-8.

19. Barreto MS, Passos VMA, Firmo JOA, Guerra HL, Vidigal PG, Lima-Costa MFF. Hypertension and clustering of cardiovascular risk factors in a community in southeast Brazil — the Bambuí health and ageing study. Arq Bras Cardiol. 2001;77(6):576-81.

20. Ministério da Saúde, Centro Nacional de Epidemiologia. Projeto monitoramento das doenças crônicas não transmissíveis (MONIT).
Relatório de pesquisa apresentado ao CENEPI. Brasília: Fundação Nacional de Saúde; 2000.

21. Alcohol drinking patterns by gender, ethnicity, and social class in Bahia, Brazil. Rev Saude Publica. 2004;38:(1):45-54.

22. O'Brien E, Waeber B, Parati G, Staessen J, Myers MG. Blood pressure measuring devices: recommendations of the European Society of Hypertension. Br Med J. 2001;322(7285): 531-6.

23. Schieri R, Oliveira MC, Pereira RA. High prevalence of hypertension among black and mulatto women in a Brazilian survey. Ethn Dis. 2001;11(3):412-8.

24. Lessa I. Hipertensão arterial. Em: Brasil, Ministério da Saúde, Secretaria de Políticas de Saúde. Manual de doenças mais importantes, por razões étnicas, na população brasileira afro-descendente. Ministério da Saúde, Secretaria de Políticas de Saúde; 2001. Pp. 43-53.

25. Mokdad AH, Ford ES, Bowman BH, Nelson DE, Engelgan MM, Vinicor F, et al. Diabetes trends in the United States, 1990-1998. Diabetes Care. 2000;23(9):1278-83.

Manuscrito recebido em 25 de julho de 2003. Aceito em versão revisada em 26 de março de 2004

ABSTRACT Objective. To estimate the frequency of modifiable cardiovascular risk factors, with and without inclusion of arterial hypertension, occurring simultaneously in a raciallymixed population.

\section{Clustering of modifiable cardiovascular risk factors in adults living in Salvador (BA), Brazil}

Method. A cross-sectional study was carried out with 1298 adults aged $\geq 20$ years in the city of Salvador, Brazil, in 2000. Eight modifiable cardiovascular risk factors were assessed, in any combination: total cholesterol $\geq 240 \mathrm{mg} / \mathrm{dL}$; high density-lipoprotein cholesterol (HDL-c) $<40 \mathrm{mg} / \mathrm{dL}$; triglycerides $\geq 200 \mathrm{mg} / \mathrm{dL}$; glycemia $\geq 126 \mathrm{mg} / \mathrm{dL}+$ wellcontrolled diabetes; body mass index $\geq 25 \mathrm{~kg} / \mathrm{m}^{2}$, waist $\geq 102 \mathrm{~cm}$ for males and $\geq 88 \mathrm{~cm}$ for females, smoking and alcoholism. The results were stratified according to the number of simultaneous risk factors (zero to five or more and two or more risk factors). The data were analyzed in terms of estimated proportions and $95 \%$ confidence intervals $(95 \% \mathrm{CI})$, with and without the inclusion of arterial hypertension (VI Joint National Committee on Prevention, Detection, Evaluation, and Treatment of High Blood Pressure [JNC-VI], United States of America), ratio of proportions and chi-square for proportions as a measure of association.

Results. Among men (41.4\% of participants), 7.5\% (95\%CI: 2.5 to 9.7) did not present risk factors; $68.8 \%$ (95\%CI: 65.0 to 72.8 ) presented two or more risk factors, not including hypertension. After inclusion of hypertension, 73.4\% (95\%CI: 69.7 to 77.1) presented two or more risk factors. Among women, $11.6 \%$ did not present risk factors. The presence of two or more risk factors, not including hypertension, was observed in $67.7 \%$ (95\%CI: 64.8 to 71.4 ). After inclusion of hypertension, $71.7 \%$ (95\%CI: 68.5 to 74.9$)$ of the women presented two or more risk factors. Significant differences were observed for the presence of two or more risk factors in men with not more than 4 years of schooling vs. 5 to less than 11 years of schooling $(P<0.05)$; in women with not more than 4 years of schooling vs. 5 to less than 11 years of schooling; in women with not more than 4 years of schooling vs. 11 or more years of schooling $(P<0.01)$; and in black vs. white women $(P<0.01)$.

Conclusions. The high proportion of clustering cardiovascular risk factors in Salvador, with or without hypertension, especially in the population with little schooling and in black individuals, suggests the need for broad social strategies to reduce social inequality, promote health, and facilitate the treatment of cardiovascular risk factors. 